\title{
Fas Receptor Modulates Lineage Commitment and Stemness of Mouse Neural Stem Cells
}

\author{
Julia Knight ${ }^{1}$, Charles Hackett ${ }^{2}$, John Soltys ${ }^{2}$, Yang Mao-Draayer ${ }^{2}$ \\ ${ }^{1}$ Neuroscience Graduate Program, University of Vermont Burlington, USA; ${ }^{2}$ Neurology Department, Fletcher Allen Health Care, \\ Burlington, USA. \\ Email: yang.mao-draayer@uvm.edu
}

Received February $1^{\text {st }}, 2011$; revised February $25^{\text {th }}, 2011$; accepted March $15^{\text {th }}, 2011$.

\begin{abstract}
Although transplanted neural stem/progenitor cells (NPCs) can ameliorate disease course in animal models of central nervous system inflammatory and neurodegenerative diseases, little is known about the regulation of NPC differentiation and proliferation. The Fas receptor, a member of the tumor necrosis factor (TNF) superfamily, has recently been shown to be important in NPC survival and immunoregulatory functions. We were interested in further investigating this system utilizing NPCs isolated from Fas-deficient (lpr) mutant mice. We found that lpr NPCs have increased survival and decreased proliferation. Additionally, RT-qPCR, confocal microscopy, and flow cytometry surface staining reveal that lpr NPCs have a significantly more robust differentiation to neuronal and oligoprogenitor cell lineages as compared to wild-type (wt) NPCs. These effects correlated with an upregulation of three of the major fate specification modulators in lpr NPCs: sonic hedgehog (Shh), slit homolog 2 (Slit2), and noggin. These data indicate Fas plays an important role in determining the stemness and differentiation fate of NPCs. Additionally, our research reveals a novel connection between Fas and major modulators of NPC differentiation-Shh, Noggin, and Slit2. This is the first indication of a possible link between Fas and these particular signaling molecules that control neuronal fate specification. Therefore, our results suggest Fas is a novel target for controlling the development of neurons versus mature oligodendrocytes.
\end{abstract}

Keywords: Fas, Neural Stem/Progenitor Cell, Differentiation

\section{Introduction}

There is a critical need for developing direct neuroregenerative therapies for demyelinating diseases, including multiple sclerosis (MS). Currently MS treatments are limited to peripheral immunoregulation and are ineffective in later, progressively neurodegenerative stages of the disease. Neural stem/progenitor cell (NPC) therapies offer a potentially powerful treatment approach for the chronic neurodegeneration that occurs in these later stages [1]. The two main experimental modalities for NPC therapy are exogenous transplantation and activation/recruitment of the endogenous adult NPC compartment [2]. In either method, differentiation and integration of NPCs to enhance central nervous system (CNS) repair is the most sought after outcome. Though exogenous application of NPC has shown significant recovery in animal models of MS (experimental autoimmune encephalomyelitis), the functional integration and terminal differentiation of transplanted cells is extremely rare [1,
3]. The signaling pathways that control NPC survival and differentiation are not well understood; therefore, elucidating these complex pathways is paramount for advancing this therapeutic approach.

The Fas receptor (hereafter referred to as "Fas")- - a member of the TNF receptor superfamily - has emerged as a major modulator of NPC biology. Previous knowledge limited this receptor to cell-death related roles: in the immune system controlling homeostasis by inducing T-cell death [4], and in the CNS mediating programmed death during embryologic development $[5,6]$. However, Fas is now recognized as a mediator of diverse, cell-dependent mechanisms. Namely, activation of Fas via its cognant ligand (FasL) promotes survival in NPCs $[7,8]$. Furthermore, Fas activation induces neurogenesis and neurite outgrowth $[8,9]$. These recent findings suggest that Fas plays a complex role in mediating NPC fate, and modulation of this pathway may be a crucial target for future therapies.

In this study, we utilized NPCs isolated from the brains 
of Fas-deficient $(l p r)$ mutant mice to further investigate the role of Fas in mediating NPC fate. Here we report that $l p r$ NPCs exhibit increased survival, increased neuronal specification, and higher populations of oligoprogenitor cells (OPCs) compared to wild type (wt) NPCs. Consistent with a more committed progenitor phenotype, lpr NPCs show deficiency in mitogenic capacity. We have identified the signaling molecules sonic hedgehog (Shh), Slit2, Hes1, and Noggin as probable mediators of these phenomena.

\section{Results}

\section{1. $L p r$ NPCs do not Express Fas}

In order to confirm that $l p r$ NPCs lack Fas, we stained NPCs with a Fas-specific phycoerythrin (PE)-conjugated antibody (or isotype control antibody). Flow cytometric analysis demonstrates high levels of Fas surface expression in wt NPCs, and no Fas expression in lpr NPCs (Figure 1).

\subsection{Fas-Deficient NPCs Have Stunted Proliferation and Neurosphere Formation}

Proliferation in response to mitogens and the ability to self-renew are defining properties of NPCs. All proliferation experiments were performed in the presence of epidermal growth factor (EGF) and fibroblast growth factor (FGF). A bromodeoxyuridine (BrDU) assay was used to determine the effects of Fas deficency on NPC proliferation. Lpr NPCs showed significantly less BrDU incorporation over 16vhours in complete media compared to $\mathrm{wt}$ NPCs. Lpr NPCs had 18\% \pm 1.6 less proliferation compared to wt (Figure 2(a)).

Additionally, neurosphere growth in $l p r$ NPCs was abrogated compared to wt NPCs (Figure 2(b)). We performed the neurosphere assay to quantify this difference. In this assay, size of the neurosphere is an indicator of mitogenic or proliferative capacity, while neurosphere number is an estimate of the absolute total number of putative stem cells [10]. Over three subsequent passages, lpr NPCs developed significantly smaller neurospheres than wt (Figure 2(c)). While, the total number of neurospheres remained the same (Figure 2(d)).

To be certain the abrogated proliferative capacity of $l p r$ NPCs was not due to altered apoptosis profiles, we also performed UV Live-Dead dye (labeling dead cells) and annexin (labeling early apoptotic cells) double-staining on NPCs growing in the presence of growth factors (EGF and FGF, similar to proliferation experiments). Four days post-cell plating, there was no difference in the amount of dead and dying cells in $l p r$ and wt cultures (Figures 2(e) and (f)). Quantification of the data showed no significant difference between the percentage of live/

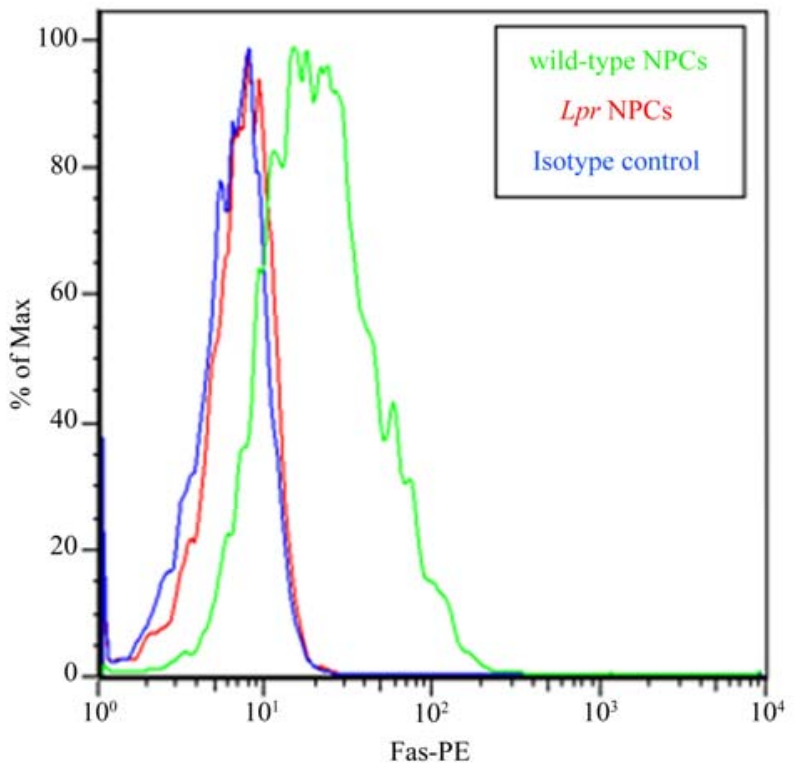

Figure 1. Lpr NPCs lacks Fas. Lpr mice have a large insertion in the Fas gene which results in improper splicing and absence of the protein. We confirmed absence of Fas in $l p r$ NPC using a phycoerythrin (PE)-conjugated anti-Fas antibody. Background staining was assessed using an isotype control IgG antibody (blue line). Unlike wild-type NPCs (green line), Ipr NPCs (red line) do not express Fas (+peak completely overlaps with isotype control). Y-axis represents $\%$ of maximum staining intensity (arbitray units) while the $\mathrm{X}$-axis represents the voltage of positive $\mathrm{PE}$ flurophore staining.

healthy cells in $\operatorname{lpr}(97 \% \pm 2.3)$ and wt $(95 \% \pm 2.6)$ cells. Therefore, the decrease in proliferation cannot be attributed to increased cell death.

In summary, Fas deficient NPCs are deficient in proliferative properties, evidenced by decreased BrDU incorporation and smaller sphere size, but Fas expression has no impact on the total number of stem cells (number of neurospheres).

\subsection{Enhanced Survival of $L p r$ NPCs in Growth Factor-Free Conditions}

The ability of NPCs to survive in the absence of growth factors (EGF, FGF) is a fundamental issue for transplantation models. If NPCs are unable to survive after transplantation due to inadequate levels of growth factors, their beneficial effects are negated. Normally, upon growth factor withdrawal in vitro, a large percentage of NPCs die [7]. We observed significantly higher survival in $l p r$ NPCs (42\%) compared to wt NPCs (25\%) after 48 hours in minimal media (no EGF or FGF) using UV/Annexin staining (described above). Therefore, the percentage of live/healthy cells in $l p r$ cultures after growth factor withdrawal is nearly doubled when compared to wt NPCs 

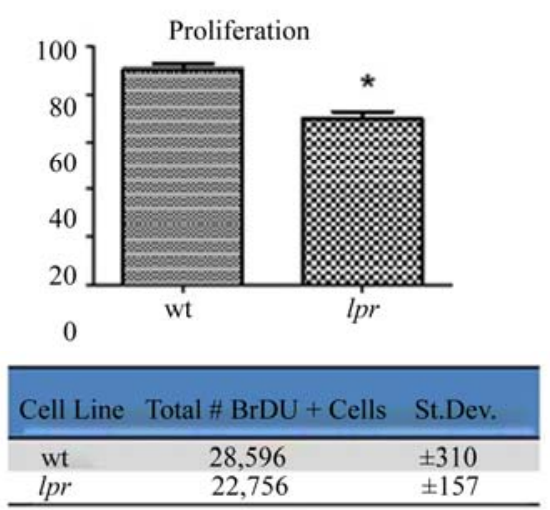

(a)

Sphere Renewal Capacity

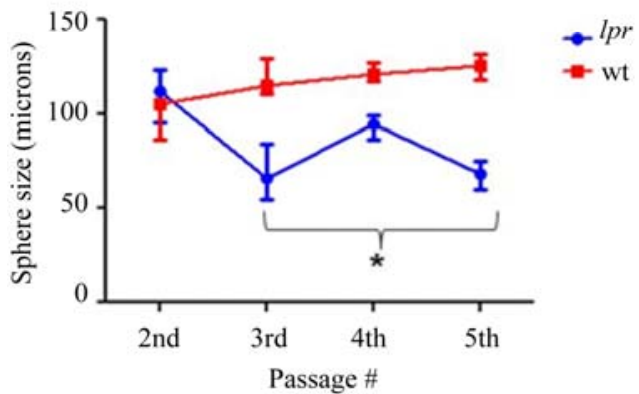

(c)

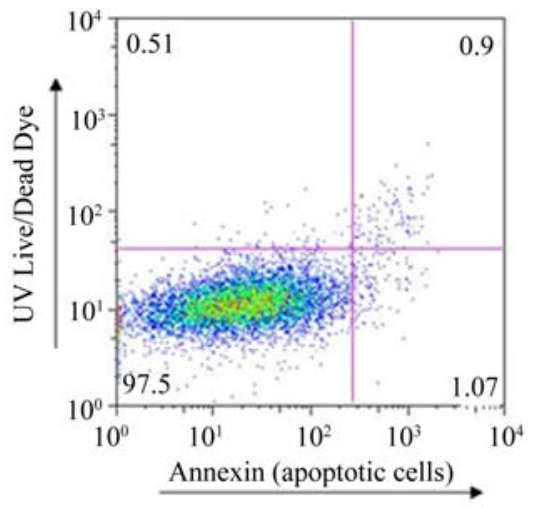

(e)
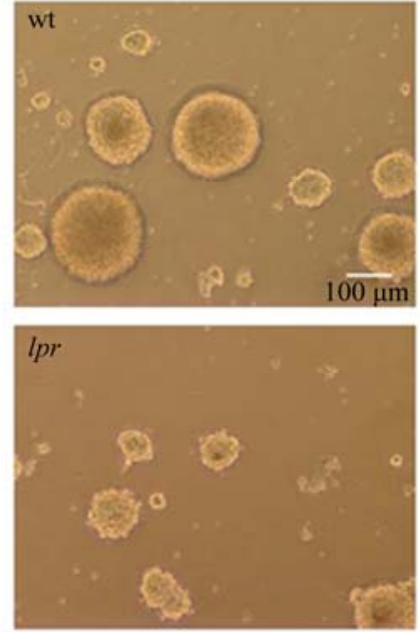

(b)

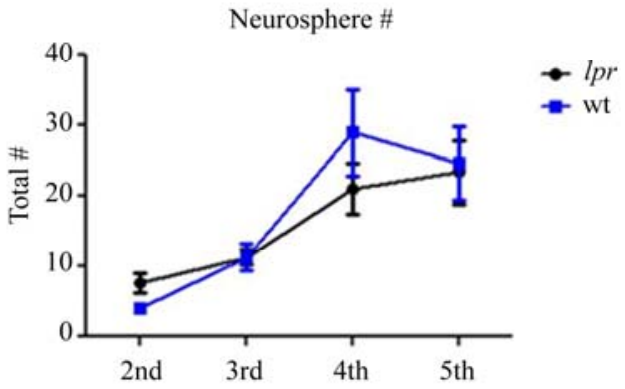

(d)

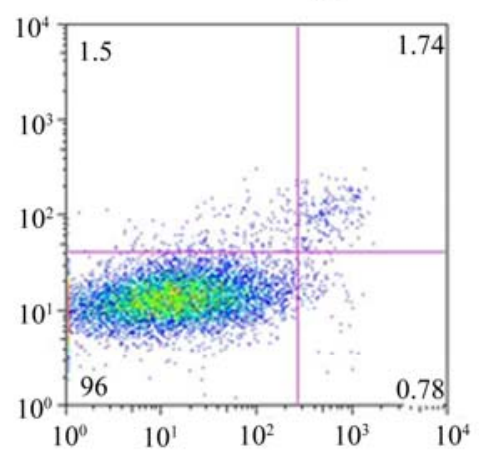

(f)

Figure 2. Abrogated proliferation and sphere growth in lpr NPCs. (a-f) All experiments performed in complete media (presence of EGF and FGF). (a) Cells growing in medium were pulsed overnight with BrDU. Proliferating cells were detected using a FITC-conjugated anti-BrDU antibody $(* p<0.05, n=4)$. Both percent positive (top) and average total number of BrDU positive cells (bottom) are shown. The total number of BrDU positive cells is out of 30,000 total events detected by the flow cytometer; (b) light microscope pictures of 7 day old tertiary neurospheres growing in complete media; (c) and (d) Neurosphere self-renewal assay: sphere size and number was quantified over 4 passages starting with passage 2 cells. Cells were dissociated and re-plated at each passage (i.e. every 7 days). $n=6$ separate wells from 2 independent experiments; (c) Neurosphere size quantification using Neurolucida ${ }^{\circledR}$ software. $L p r$ neurospheres were significantly smaller than wild-type at passage number 3, 4, and 5 (* $\left.{ }^{*}<0.05\right)$; (d) Quantification of neurosphere number. Shown is the average number within 20X phase microscope images (as shown in (b)); (e) and (f) representative dot plots of cells growing in complete media for four days. Y-axis represents dead cells (staining positive for UV Live/Dead dye) and X-axis represents apoptotic cells (staining positive for annexin-AlexaFluor647). The majority of cells are live/healthy (lower left quadrant), with no differences between $\operatorname{lpr}(\mathrm{e})$ and wt (f) samples. 
(Figures 3(a) and (b)).

\subsection{Lpr NPCs Exhibit Enhanced Differentiation to Neuronal and Oligoprogenitor Cell Lineages}

One distinguishing feature of NPCs is their capacity to differentiate into the three neuroectodermal lineages. We compared $l p r$ and wt NPC lineage marker RNA expression for astrocytes (glial fibrillary acidic protein (GFAP), glutamate aspartate transporter (GLAST)), OPCs (platelet-derived growth factor receptor- $\alpha(\operatorname{PDGFR} \alpha))$ and neurons ( $\beta$ III-tubulin, Doublecortin (Dcx)) using RTqPCR. After two days of differentiation, lpr NPCs expressed significantly higher levels of OPC and neuronal markers compared to wt NPCs (Figure 4(a)). This trend maintained statistical significance after seven days in differentiating media (Figure 4(b)).

We confirmed the enhanced OPC phenotype in $l p r$ NPCs by staining for A2B5, a putative OPC cell surface marker, after two days of differentiation. Flow cytometric analysis revealed $60 \%$ of $l p r$ NPCs were positive for A2B5, while only $20 \%$ of wt NPCs were A2B5-positive (Figures 5(a) and (b)).

NPC cell morphology was also confirmed at the protein level using immunocytochemistry. Staining for $\beta$ IIItubulin and PDGFR $\alpha$ corroborated mRNA expression levels, as the percentage of neuronal cells ( $\beta$ III-tubulin + ) and OPCs (PDGFR $\alpha+$ ) in lpr cultures was double that found in wt (Figures 5(c) and (d)).

Altogether, these data indicate that the absence of Fas enhances NPC differentiation to the neuronal and OPC lineages at both the genomic and protein level.

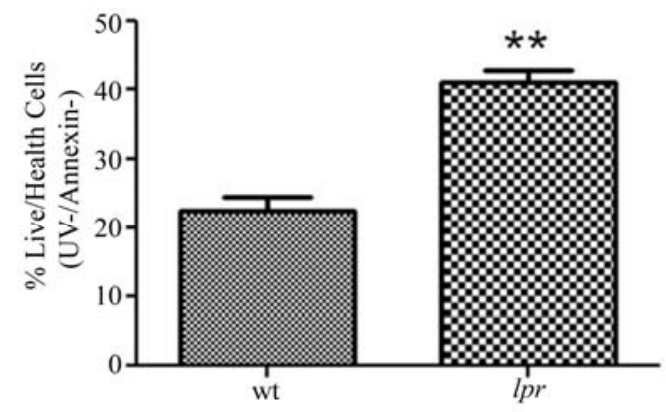

(a)

\begin{tabular}{ccc}
\hline Cell Line & Total \# Viable Cells & St.Dev. \\
\hline wt & 4,244 & \pm 405 \\
\hline$l p r$ & 10,597 & \pm 813 \\
\hline
\end{tabular}

(c)

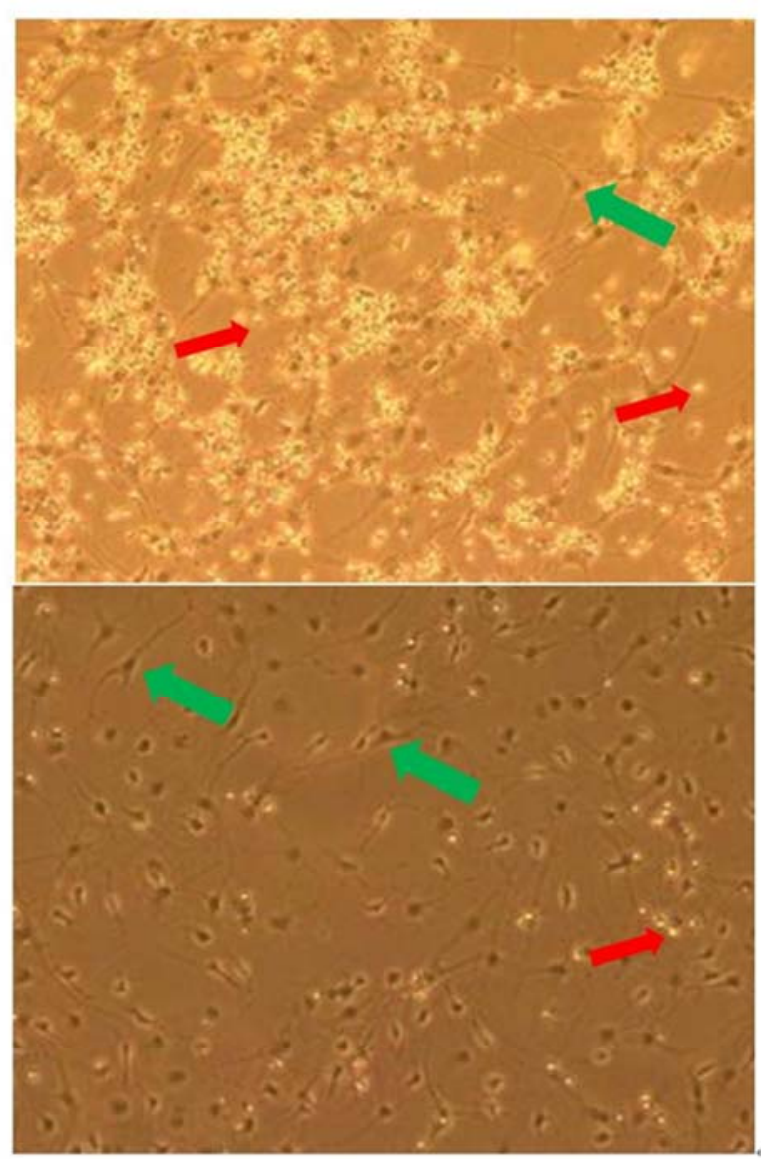

(b)

Figure 3. Fas-deficient NPCs have increased survival in the absence of growth factors. Cultures were switched to minimal media (no EGF or FGF) for $48 \mathrm{hr}$ and then the apoptosis (Annexin) and death (UV dye) assay was performed and analyzed using flow cytometry. (a) Plotted are the percentages of healthy cells, which are negative for either stain. $n=5$ (\#independent experiments), error bars represent SEM. In representative phase microscope images (b), bright and floating bodies represent dead cells (indicated by smaller, red arrows) while attached cells with processes represent live/healthy cells (indicated by larger, green arrows); (c) Average number of total viable cells in each culture $(n=5)$. The total number of viable $(\mathrm{UV}-/ \mathrm{Annexin}-)$ cells is out of 20,000 total events detected by the flow cytometer. $* * p<0.01$. 


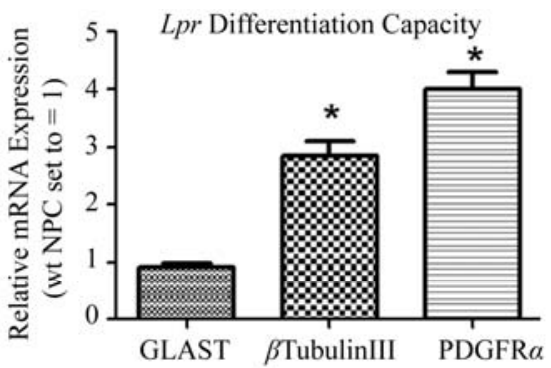

(a)

\begin{tabular}{|c|c|c|}
\hline Lineage Marker & 2 days & 7 days \\
\hline Doublecortin & $+4.98 \pm 1.70$ & $+5.12 \pm 1.29$ \\
\hline$\beta$ III-tubulin & $2.85 \pm 0.50$ & $1.8 \pm 0.15$ \\
\hline PDGFR $\alpha$ & $4.00 \pm 0.60$ & $2.8 \pm 0.55$ \\
\hline GLAST & No change & No change \\
\hline GFAP & No change & No change \\
\hline
\end{tabular}

(b)

Figure 4. Ipr NPCs are more committed to oligo- and neuronal lineages. (a) Spontaneous differentiation of $l p r$ cells compared to wt after 2 days in minimal media; (b) summary of lineage marker results at 2 days (minimal media) and 7 days $(0.1 \%$ FCS). Fold changes are $l p r$ expression levels relative to wt. Each represented fold difference reached statistical significance $(p<0.05, n=5$ independent experiments). Fold changes calculated using $2^{-\Delta \Delta C t}$ method with $\beta$-actin as the endogenous control gene and wt NPC samples as the calibrator (mRNA expression levels set to equal 1).

\subsection{Lpr NPCs Exhibit Altered Levels of Neuronal Fate Specification Genes, Including Shh}

In search of possible mechanisms and pathways controlling the $l p r$ phenotype, we ran a "Neurogenesis and Neural Stem Cell" (SA Biosciences ${ }^{\mathrm{TM}}$ ) PCR array using RNA isolated from wt or $l p r$ NPCs after 7 days of differentiation. Of the $>80$ genes tested, four were downregulated (apolipoprotein E (Apoe), bone morphogenetic protein 2 (BMP2), FGF2, S100 calcium binding protein A6 (S100a6)) and 6 were up-regulated ( $\alpha$-filamin (Flna), hairy and enhancer of split 1 (Hes1), Neurod1, Noggin, neuronal pentraxin 1 ( $\mathrm{NptX1})$, Shh) in lpr compared to wt (Figure 6(a)). Neurod1, Noggin, and Nptx1 are all genes known to be involved in regulating neuronal fate specification. Shh is a major modulator of both oligodendrocyte and neuronal differentiation pathways. The Shh antagonist BMP2 is down-regulated in $l p r$ NPCs $(-3.87$ fold, $p<0.0012)$; further indicating Shh pathway alterations as a probable mechanism for the altered $l p r$ phenotype.

Upregulation of Hes1, Noggin, and Shh was confirmed using RT-qPCR (Figure 6(b)). To further evaluate the involvement of Shh in the lpr phenotype, we also tested expression levels of Gli1, a major regulator and target of Shh signaling. Gli1 was up-regulated 3.86-fold $( \pm 0.55)$ in lpr NPCs compared to wt.

\section{Discussion}

There are currently no efficacious treatments for devastating neurodegenerative diseases including Alzheimer's and MS. Therefore, there is a pressing need to develop therapeutics that exhibit neuroprotective effects by acting directly on the CNS. NPC therapies represent a novel and promising avenue for developing neuroregenerative treatments $[2,11]$. However, all stem cell therapies currently in development ultimately fail in that they are unable to completely repair lost or damaged CNS tissue $[12,13]$. If endogenous or transplanted NPCs could be activated to terminally differentiate, CNS damage and consequent long-term disability could be minimized or even reversed. Therefore, determining modulators of NPC differentation and their mechanisms of action is critical to advancing therapies for neurogenenerative disease.

By utilizing a Fas-deficient mutant NPC cell line (lpr), we were able to determine the significance of Fas-signaling in NPC survival, self-renewal capacity, and differentiation into the three neuroectodermal lineages. $\mathrm{Lpr}$ mice have a retroviral insertion of poly(A) adenylation signal repeats in the gene for Fas [4]. This large insertion results in improper splicing and truncation of Fas mRNA so that the cells are devoid of protein expression. Our study using NPCs isolated from lpr mice is novel in that there has not been detailed characterization of how lack of Fas affects NPC stemness and differentiation ex vivo.

We showed that $l p r$ NPCs have dramatically decreased cell death upon withdrawal of growth factors - a condition that normally induces both significant cell death and spontaneous differentiation to astrocytes, neurons, and sparse oligodendrocytes. The significance of this discovery is two-fold. First, this suggests that Fas manipulation may represent a feasible solution to promote NPC viability in harsh environments, including that introduced in exogenous stem cell transplantation paradigms. Second, enhanced survival of NPCs could allow for enhanced neuroprotective and immunoregulatory capacity in disease models. We hypothesize that extended survival of lpr NPCs in the absence of growth factors increases their capacity for terminal OPC and neuronal differentiation, as wild-type NPCs are more likely to die before activating differentiation pathways.

This hypothesis is further supported by our data indicating that $l p r$ NPCs have an increased expression of oligoprogenitor cell (OPC) lineage markers and enhanced differentiation into mature neurons. Up-regulated 


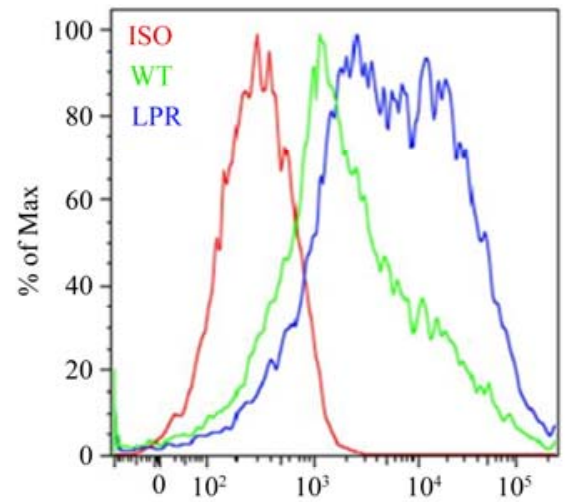

(a)
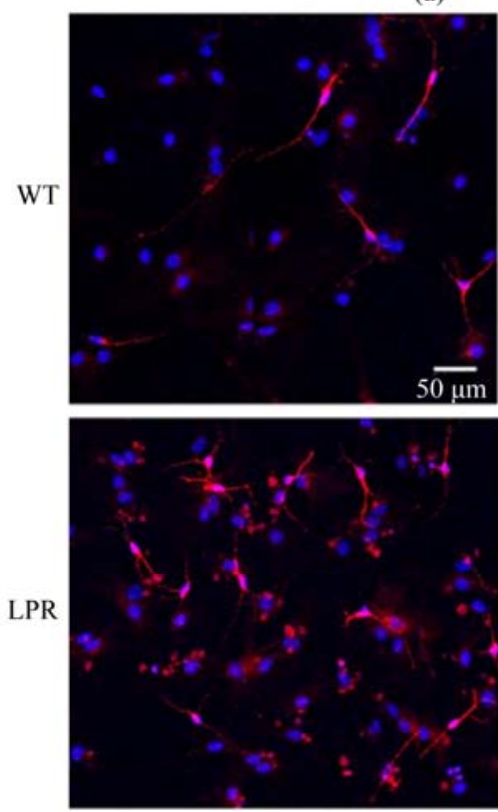

$\beta$ III-tubulin
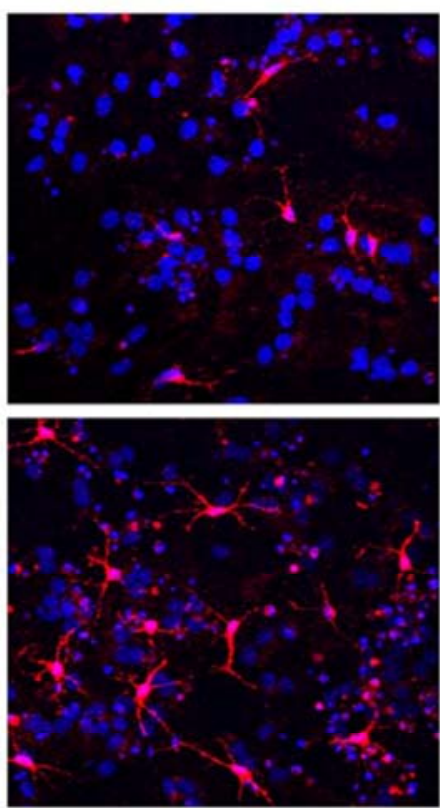

PDGFR $\alpha$

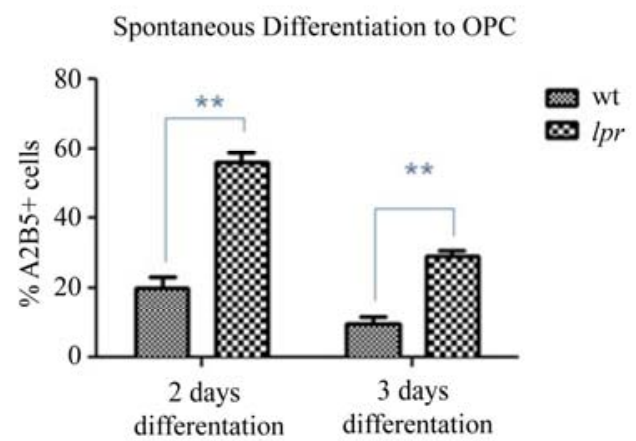

(b)

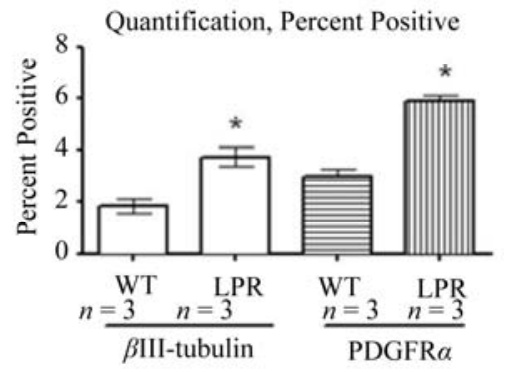

(d)

(c)

Figure 5. LPR NPCs have enhanced differentiation into Neuronal and OPC lineages. (a) Representative fluorescence histogram for A2B5 positivity after 2 days in minimal media (red = isotype control [ISO]); (b) Quantification of A2B5 flow data ( $n$ $=4$ independent experiments, $* * p<0.01$ ). After 3 days of differentiation (right columns), lpr NPC still expressed a significantly higher amount of A2B5, though overall levels of this marker is decreased in both cell types. (c \& d) Immunocytochemistry (ICC) was performed on cells after seven days in differentiating media; (c) Differentiation of wt (top panels) and $l p r$ (bottom panels) into neurons ( $\beta$ III-tubulin+ cells, left panels) and OPCs (PDGFR $\alpha+$ cells, right panels); (d) Quantification of ICC data using Image $J$ software. Percent positive = \#lineage marker+/total \# of Hoechst+. 12 - 14 serial photos were taken from 3 separate coverslips. * $p<0.05, n=3$.

OPC marker expression in lpr compared to wt NPCs was detected by analyzing mRNA levels using RT-qPCR and confirmed using both flow cytometry surface staining (A2B5) and confocal microscopy to confirm the OPC phenotype (PDGFR $\alpha$ ).

Along with elevated levels of OPC markers, lpr NPCs have elevated levels of neuronal-specific lineage markers (BIII-tubulin, doublecortin) both at early (two days) and late (seven days) differentiation time points. In addition, the astrocyte-specific marker GFAP remains unchanged.
These data indicate a preferential neuronal-fate specification in NPCs lacking Fas. Corroborating these data, $l p r$ NPCs show significantly increased expression of genes known to control neuronal lineage specification and maturation: Noggin, Neurod1, and Nptx1. We identify Shh, Slit2, and Hes1 signaling as putative upstream regulators of this neuronally-biased phenotype. Hes 1 is a member of the basic helix-loop-helix (bHLH) transcriptional repressors that regulate cell proliferation and differentiation during embryogenesis. Levels of Hes 1 mo- 


\begin{tabular}{|c|c|c|}
\hline Gene & $\begin{array}{c}\text { Fold-Regulation } \\
(l p r / w t)\end{array}$ & $p$-value \\
\hline Apoe & -2.34 & 0.0058 \\
\hline BMP2 & -3.87 & 0.0012 \\
\hline Fgf2 & -2.85 & 0.0042 \\
\hline Flna & 2.35 & 0.0112 \\
\hline Hes1 & 6.09 & 0.0017 \\
\hline Neurod1 & 3.55 & 0.0126 \\
\hline Nog & 4.32 & 0.0125 \\
\hline Nptx1 & 2.51 & 0.0109 \\
\hline S100a6 & -2.75 & 0.0124 \\
\hline Shh & 23.4 & 0.0172 \\
\hline Slit2 & 10.3 & 0.0285 \\
\hline
\end{tabular}

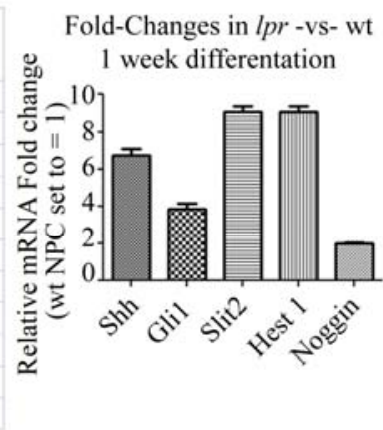

(a) (b)

Figure 6. Upregulation of Shh and neuronal specification genes in lpr NPCs. (a) Shown are genes with significant mRNA fold-changes (out of $>80$ tested, SA Biosciences ${ }^{\text {TM }}$ "Neurogenesis and Neural Stem Cell" PCR Array) in lpr NPCs compared to wt NPCs after seven days in differentiation medium. Down-regulated genes (red font) have been converted to whole numbers via $1 /$ [fractional fold change]; (b) RT-qPCR confirmation of gene upregulation in lpr NPCs after one week in differentiation media. Data are based on 4 biological replicates $(n=4)$, all fold changes are significantly above levels in wt cells $(p<0.01)$.

dulate differentiation of ES cells to neuronal and mesodermal cells and oscillating levels contribute to heterogeneous responses from these cells [14]. Slit2 acts through the Roundabout (Robo) receptor and is involved in axon guidance $[15,16]$ and neuronal migration [17]. Shh is a critical gene involved in patterning during embryogenesis and regulates NPC proliferation and neurogenesis $[18,19]$. Since the absence of functional Fas in our culture system enhanced neuronal differentiation, it is possible that active Fas in wt NPCs provides inhibitory signals against this lineage development. Whether these inhibitory signals act upon the candidate genes identified here requires further study.

Previously, we [7] and others [8] have shown that activation of Fas via FasL enhances NPC survival. Additionally, in vivo overexpression of FasL increases subventricular zone neurogenesis. An explanation for these seemingly contradictory results is that exposure to FasL in NPCs enhances both survival and neurogenic pathways [7,8]; however, the presence of a functional Fas system in the absence of activating signals (FasL) inhibits these pathways. The latter is indicated by our current study since endogenous FasL levels are extremely low in our culture system [4] and wt NPCs have decreased survival and differentiation compared to lpr NPCs-presumably because unactived Fas serves an inhibitory role in these phenomena. This suggests a dynamic role for Fas in both NPC fate specification and survival that is dependent on the presence or absence of FasL.

Taken together, we propose the following paradigm for the role of Fas in NPCs. Fas targeting leads to two

unique physiological processes: first, Fas ablation promotes NPC survival in deprived-environments and second, Fas ablation promotes increased neuronal and oligo differentiation. Coupled together, Fas manipulation may produce a viable means of exogenous stem cell transplantation by overcoming the challenge of cell survival under harsh transplantation conditions, while promoting complete integration of NPCs into functional neuronal circuits by stimulation of NPC differentiation. The Shh signaling pathway, or other molecules identified here, may be a useful target in promoting this response. This paradigm offers novel candidates deserving further investigation because of their potential to advance NPC transplantation therapies.

\section{Conclusions}

We have identified Fas as an important modulator of NPC survival, differentiation, and proliferation. This study is the first to demonstrate a putative mechanistic link between Fas and Shh signaling. Overall, we provide important insight into understanding the elusive mechanisms that dictate NPC fate-determination, an important step in improving NPC-based therapies for neurodegenerative disorders.

\section{Experimental Procedure}

\subsection{NPC Isolation and Culture}

NPCs were isolated from whole brains dissected from 4-day postnatal wild-type C57/BL6 or lpr mutant mice (C57/BL6 background). Brains were processed using NeuroCult $\circledast$ Enzymatic Dissociation Kit for CNS Tissue (Stem Cell Technologies). Resulting single-cell suspension was plated in EGF and FGF (both $10 \mathrm{ng} / \mathrm{mL}$ ) containing complete media (Gibco®Neural Basal Media supplemented with B27 and 1:1:2). Neurospheres were chemically dissociated (Kit, Stem Cell Technologies) into a single-cell suspension and plated on poly-D Lysine and laminin (both Sigma) coated Nunc ${ }^{\circledR}$ flasks or plates for passaging and experimentation. Cells were plated at a density of 16,000 cells $/ \mathrm{mL}$ media and grown to confluency (3 - 4 days) before experimentation or switching to minimal media. All experiments were performed on passage-matched wt or lpr NPC lines and only cells with passage numbers between 3 and 6 were used. Proliferation studies were performed in EGF and FGF containing media, cell death studies and early differentiation timepoints were performed in minimal media (Neural Basal Media with only B27 and 1:1:2 [penicillin/streptomycin: L-glutamine: glucose]), and extended differentiation experiments were performed in minimal media containing $0.2 \%$ FCS (to prevent extensive cell death in the absence of growth factors). All cells incubated in $37^{\circ} \mathrm{C} / 5 \%$ $\mathrm{CO}_{2}$ conditions. 


\subsection{Flow Cytometry}

Cells were lifted using $0.05 \%$ trypsin/EDTA (Gibco) at $37^{\circ} \mathrm{C}$. Trypsin enzyme was stopped using $20 \%$ FCS/ neural basal media after 7 mins. Cells were placed in Falcon ${ }^{\circledR}$ tubes (\#352058) and then rinsed with 1XPBS and spun at $1400 \mathrm{rpm}$ before proceeding with staining. All stains were added to a $100 \mu \mathrm{L}$ volume cell suspension containing approximately $1 \times 10^{6}$ cells.

Fas receptor stain: Samples blocked with hamster IgG $(50 \mathrm{ug} / \mathrm{mL})$. Samples stained with either anti-Fas or isotype control (Hamster IgG2, $\lambda 1$ ) phycoerythrin (PE)-conjugated antibodies (1:30, BD Biosciences). All rinses and dilutions were done with $1 \% \mathrm{BSA} / 1 \mathrm{XPBS}$. All incubations performed at $4^{\circ} \mathrm{C}(15 \mathrm{~min}$ for block, $30 \mathrm{~min}$ for PE-conjugated antibodies).

A2B5 stain: biotynlated anti-A2B5 (50 uL, 1:100, BD Pharm) added to each sample. Primary antibody incubated for $30 \mathrm{~min}$ at $4^{\circ} \mathrm{C}$. After rinsing with PBS, Strepavidin-Phycoerythrin (100 uL, undiluted, BD Pharm) added to each sample and incubated for $30 \mathrm{~min}$ at $4^{\circ} \mathrm{C}$. Samples rinsed twice before analyzing on flow. Controls included: no primary and a sample of NPCs growing in the presence of EGF and FGF (to serve as a low percent differentiation negative control).

Death \& Apoptosis Assay (UV/Annexin stain): UV Live/Dead Dye ${ }^{\circledR}$ and Annexin Alexa Fluor-647 both used according to manufacturer instructions (Invitrogen). UV dye labels dead cells because of disruptions in the cell membrane. Viable cells to not stain positive for UV dye because of an intact cell membrane. Annexin binds strongly to phosphatidylserine residues that have flipped to the exterior surface of the cell, thus labeling early apoptotic cells. Briefly, $200 \mu \mathrm{L}$ of diluted UV dye $(2 \mu \mathrm{L}$ per $\mathrm{mL} 1 \times \mathrm{PBS}$ ) was added to cell suspension containing 10,000 cells $/ \mu \mathrm{L}$. Cells were incubated on ice, in the dark, for $30 \mathrm{~min}$. After cells with $1 \times \mathrm{PBS}$ and aspirating the supernatant, $100 \mathrm{uL}$ of diluted Annexin $(1 \mu \mathrm{L}$ per 400 $\mu \mathrm{L}$ HEPES buffer) was added to cells. Cells grown in complete media (growth factors present) were used as a negative control because $>90 \%$ of cells in these cultures are live/healthy. Cells briefly treated with $3 \%$ paraformaldehyde were used as positive controls because $>90 \%$ of cells are dead/dying.

Cell Proliferation: BrDU assay performed according to manufacturer instructions (FITC Detection BrdU Flow Cytometry Assay Kit, BD Biosciences). Briefly, cells were first pulsed overnight (16 hours) with BrDU and subsequently fixed and stained with a FITC-conjugated anti-BrDU antibody for flow analysis. Cells grown in minimal media (no growth factors) were used as a negative control because $<10 \%$ of cells grown in these conditions will stain positive for BrDU incorporation.
Analysis: flow cytometry analysis was performed by a BD LSRII flow cytometer equipped with 3 lasers (488, helium neon, and UV) and data were analyzed with FlowJo software, respectively.

\subsection{Neurosphere Self-Renewal Assay}

Single-cell suspensions were plated in uncoated 24-well plates (seeding density of 100,000 cells $/ \mathrm{mL}$ ) using NPC media containing EGF and FGF. After 7 days, 2-3 pictures of random frames from each well ( 3 wells per cell type) were taken at $20 \mathrm{X}$ using a phase-contrast microscope. After taking pictures, neurospheres were dissociated using Neurocult ${ }^{\circledR}$ Chemical Dissociation Kit and then filtered through a $40 \mu \mathrm{m}$ nylon cell strainer (BD Falcon $^{\mathrm{TM}}$ ). Cells were then counted, plated as described above, and grown for 7 days before the next passage time point was recorded (this same process completed four total times over four weeks). Exact cell circumferences were measured using Neurolucida software.

\subsection{Immunocytochemistry}

Cells were plated in 24-well plates with poly-D/Laminin coated coverslips and grown to confluency. At this point, cells were rinsed and allowed to differentiate 1 week in minimal media containing $0.2 \%$ FCS. For all differentiation markers, 3 coverslips were scored per cell line and repeated for at least 3 biological replicates.

Stains performed as follows: Cells fixed with Zamboni's fixative and blocked $(10 \% \mathrm{HS} / 0.1 \%$ Triton $/ 0.02 \%$ Azide in $1 \times$ PBS) for 30 min. Block for PDGFR $\alpha$ stain did not include triton. Primaries incubated overnight at $4^{\circ} \mathrm{C}$, while secondary antibodies incubated for $1.5 \mathrm{hr}$ at RT. Cells rinsed $3 X$ between primary and secondary. All cells counterstained with Hoechst nuclear stain (1:2000).

The following antibodies were used: rabbit anti-BIII Tubulin (Sigma, 1:100), rabbit anti-PDGFR $\alpha$ (Santa Cruz, 1:50), rabbit anti-GFAP (Dako, 1:1000), goat anti-rabbit Cy3 (1:500, Jackson Immunoresearch).

All confocal images were acquired using the "Tile" feature on a Zeiss LSM 510 META confocal laser microscope. Cells were visualized on the left side of each cover slip at $20 \mathrm{X}$ magnification, and then the microscope acquired 16 consecutive field-of-view images in the form of a $4 \times 4$ square (referred to here as a tile). All cell scoring was performed using ImageJ software. Each tile was scored for the number of nuclei present (hoecsht positive cells) and the number of cells staining positive for the respective differentiation marker (a cell was considered positive if the cell body and projections were labeled). At least 9 tiles were acquired and tallied per slip, resulting in the total acquisition of $>75$ individual fields-of-view per coverslip. Percent positive was calculated by dividing the number of cells positive for a lineage marker ( $\beta$ III-tubu- 
lin or PDGFR $\alpha$ ) by the total number cells (hoecsht staining nuclei).

\subsection{RT-qPCR and Gene Expression Arrays}

Performed as previously described [7]. Briefly, RNA was extracted from cells using RNeasy ${ }^{\circledR}$ Mini Kit (Qiagen) according to manufacturer protocol. For RT-qPCR, cDNA was prepared using Superscript ${ }^{\circledR}$ III First-Strand Synthesis Supermix for qRT-PCR. RT-qPCR completed using Taqman ${ }^{\circledR}$ Master Mix and applied Biosystems 7500 Fast Software. Primers were purchased from Applied Biosystems (Taqman Assay on Demand).

Neurogenesis and Neural Stem Cell (PAMM-404) PCR arrays purchased from SA Biosciences ${ }^{\mathrm{TM}}$ and RNA samples were processed by UVM Cancer Center DNA Facility.

\subsection{Statistics}

Graphs were created and statistical analyses were run using GraphPad Prism software. Student's $t$-test or ANOVA with Tukey's post-test were used to determine significance ( $p$-values).

\section{Acknowledgements}

We would like to thank: Dr. Rae Nishi for helpful discussion and guidance; Dr. Jeffrey Spees and the UVM Stem Cell Core for resources and general support; Dr. Ralph Budd for graciously supplying $l p r$ mice; Dr. Karen Fortner for expertise and assistance; Dr. Issei Shimada for technical assistance and guidance; John DeWitt and Anthony Pappas for technical assistance; Timothy Hunter and colleagues for services provided by the Vermont Cancer Center DNA Analysis Facility; UVM Neuroscience Center of Biomedical Research Excellence (COBRE) for resources and technical support; and the UVM Microscopy and Imaging Center for resources and Marilyn Wadsworth for technical assistance and training. The project described was supported by NIH Grant Number P20 RR16435 from the COBRE Program of the National Center for Research Resources.

\section{Authors' Contributions}

JK - study concept and design, manuscript preparation, data acquisition and analysis; $\mathrm{CH}$ - data acquisition and analysis; JS - data acquisition and analysis, manuscript revisions; YMD - study concept and design, manuscript revisions.

\section{REFERENCES}

[1] S. Pluchino and G. Martino, "The Therapeutic Use of Stem Cells for Myelin Repair in Autoimmune Demyelinating Disorders," Journal of the Neurological Sciences, Vol. 233, No. 1-2, 2005, pp. 117-119.

\section{doi:10.1016/i.jns.2005.03.026}

[2] G. Martino and S. Pluchino, "The Therapeutic Potential of Neural Stem Cells," Nature Reviews Neuroscience, Vol. 7, No. 5, 2006, pp. 395-406. doi:10.1038/nrn1908

[3] S. Pluchino, L. Zanotti, B. Rossi, E. Brambilla, L. Ottoboni, G. Salani, M. Martinello, A. Cattalini, A. Bergami, R. Furlan, et al., "Neurosphere-Derived Multipotent Precursors Promote Neuroprotection by an Immunomodulatory Mechanism," Nature, Vol. 436, No. 7048, 2005, pp. 266-271. doi:10.1038/nature03889

[4] S. Nagata and P. Golstein, "The Fas Death Factor," Science, Vol. 267, No. 5203, 1995, pp. 1449-1456. doi:10.1126/science. 7533326

[5] F. K. H. van Landeghem, U. Felderhoff-Mueser, A. Moysich, C. Stadelmann, M. Obladen, W. Brück and C. Bührer, "Fas (CD95/Apo-1)/Fas Ligand Expression in Neonates with Pontosubicular Neuron Necrosis," Pediatric Research, Vol. 51, No. 2, 2002, pp. 129-135. doi:10.1203/00006450-200202000-00003

[6] R. Nat, E. Radu, T. Regalia and L. M. Popescu, "Apoptosis in Human Embryo Development: 3. Fas-Induced Apoptosis in Brain Primary Cultures," Journal of Cellular and Molecular Medicine, Vol. 5, No. 4, 2001, pp. 417- 428. doi:10.1111/j.1582-4934.2001.tb00177.x

[7] J. Knight, E. Scharf and Y. Mao-Draayer, "Fas Activation Increases Neural Progenitor Cell Survival," Journal of Neuroscience Research, Vol. 88, No. 4, 2010, pp. 746757.

[8] N. S. Corsini, I. Sancho-Martinez, S. Laudenklos, D. Glagow, S. Kumar, E. Letellier, P. Koch, M. Teodorczyk, S. Kleber, S. Klussmann, et al., "The Death Receptor CD95 Activates Adult Neural Stem Cells for Working Memory Formation and Brain Repair," Cell Stem Cell, Vol. 5, No. 2, 2009, pp. 178-190. doi:10.1016/j.stem.2009.05.004

[9] J. Desbarats, R. B. Birge, M. Mimouni-Rongy, D. E. Weinstein, J.-S. Palerme and M. K. Newell, "Fas Engagement Induces Neurite Growth through ERK Activation and P35 Upregulation," Nature Cell Biology, Vol. 5, No. 2, 2003, pp. 118-125. doi:10.1038/ncb916

[10] S. Pluchino, L. Muzio, J. Imitola, M. Deleidi, C. Alfaro-Cervello, G. Salani, C. Porcheri, E. Brambilla, F. Cavasinni, A. Bergamaschi, et al., "Persistent Inflammation Alters the Function of the Endogenous Brain Stem Cell Compartment," Brain, Vol. 131, No. 10, 2008, pp. 2564-2578. doi:10.1093/brain/awn198

[11] R. J. M. Franklin and C. Ffrench-Constant, "Remyelination in the CNS: From Biology to Therapy," Nature Reviews Neuroscience, Vol. 9, No. 11, 2008, pp. 839-855. doi: $10.1038 / \mathrm{nrn} 2480$

[12] M. Stangel, "Neuroprotection and Neuroregeneration in Multiple Sclerosis," Journal of Neurology, Vol. 255, Supplement 6, 2008, pp. 77-81. doi:10.1007/s00415-008-6014-x

[13] W. F. Blakemore, "Regeneration and Repair in Multiple Sclerosis: The View of Experimental Pathology," Journal of the Neurological Sciences, Vol. 265, No. 1-2, 2008, pp. 


\section{1-4. doi:10.1016/i.jns.2007.03.006}

[14] T. Kobayashi, H. Mizuno, I. Imayoshi, C. Furusawa, K. Shirahige and R. Kageyama, "The Cyclic Gene Hes1 Contributes to diverse Differentiation Responses of Embryonic Stem Cells," Genes \& Development, Vol. 23, No. 16, 2009, pp. 1870-1875. doi:10.1101/gad.1823109

[15] K. Patel, J. A. Nash, A. Itoh, Z. Liu, V. Sundaresan and A. Pini, "Slit Proteins Are Not Dominant Chemorepellents for Olfactory Tract and Spinal Motor Axons," Development, Vol. 128, No. 24, 2001, pp. 5031-5037.

[16] L. Lin and O. Isacson, "Axonal Growth Regulation of Fetal and Embryonic Stem Cell-Derived Dopaminergic Neurons by Netrin-1 and Slits," Stem Cells, Vol. 24, No. 11, 2006, pp. 2504-2513. doi:10.1634/stemcells.2006-0119
[17] W. Wu, K. Wong, J. Chen, Z. Jiang, S. Dupuis, J. Y. Wu and Y. Rao, "Directional Guidance of Neuronal Migration in the Olfactory System by the Protein Slit," Nature, Vol. 400, No. 6742, 1999, pp. 331-336. doi: $10.1038 / 22477$

[18] G. Zhu, M. F. Mehler, J. Zhao, Y. S. Yu and J. A. Kessler, "Sonic Hedgehog and BMP2 Exert Opposing Actions on Proliferation and Differentiation of Embryonic Neural Progenitor Cells," Developmental Biology, Vol. 215, No. 1, 1999, pp. 118-129. doi:10.1006/dbio.1999.9431

[19] V. Palma, D. A. Lim, N. Dahmane, P. Sánchez, T. C. Brionne, C. D. Herzberg, Y. Gitton, A. Carleton, A. A1varez-Buylla and A. Ruiz i Altaba, "Sonic Hedgehog Controls Stem Cell Behavior in the Postnatal and Adult Brain," Development, Vol. 132, No. 2, 2005, pp. 335-344. doi:10.1242/dev.01567

GLAST: glial high affinity glutamate transporter PDGFR $\alpha$ : platelet-derived growth factor receptor- $\alpha$ Dcx: Doublecortin GalC: galactosylceramidase Shh: sonic hedgehog FGF: fibroblast growth factor EGF: epidermal growth factor 